Ärztliche Erfahrung beschränkt sich nicht auf medizinisches Fachwissen.

\title{
Im Wein liegt die wütende Wahrheit
}

Nach einem wahnsinnig anstrengenden Praxistag fuhr ich mit meinem Mann in eine gemütliche Weinstube in den Rheingau, mit Blick auf den Rhein, um auszuspannen.

Mit der Erholung war es erst mal nix. Am Nebentisch saß ein Mann, der immer lauter und aufgeregter auf seine Begleiterinnen einredete. Die Situation wurde schnell sehr unangenehm. Bald konnte man es im ganzen Lokal knistern spüren. Immer mehr Köpfe drehten sich zu dem Wüterich um. Da reichte es meinem Mann. „Sie gehen hier allen auf den Zeiger", fuhr er unseren Tischnachbarn plötzlich an. Ich spürte, wie mein Vegetativum ansprang. Kam es hier jetzt auch noch zur Saalschlacht?

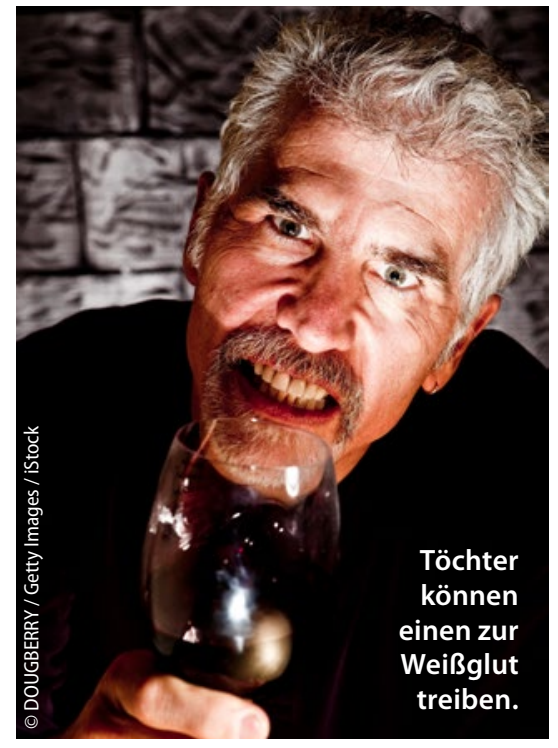

Doch das Gegenteil trat ein: Der aufgebrachte Mann entschuldigte sich, fragte aber auch gleich: „Haben Sie eine Tochter?" Es war offenbar der weibliche Nachwuchs, der ihn so aus der Fassung gebracht hatte.

Schon am nächsten Tag war mir die Episode nützlich, als die Mutter einer pubertierenden Tochter in meiner Praxis erschien. Ihr Mädchen hatte wirklich nichts ausgelassen, um die arme Mutter auf die Palme zu bringen. Ich erhob mich von meinem Platz und erzählte ihr die Geschichte aus der Weinstube. Das beruhigte die Frau und sie ging, glaube ich, singend aus der Praxis: „You never walk alone!“

Dr. Luise Hess, Darmstadt

\section{Teuflischer Blutdruck!}

_ Bei einer 48-jährigen Patientin diagnostizierte ich im Rahmen des Checkups eine Hypertonie. In dem sich anschließenden Beratungsgespräch erklärte ich ihr, das man in ihrem Fall versuchen sollte, die Lage medikamentös in den Griff zu bekommen.

Sie wollte das allerdings nicht recht wahrhaben. Sie beharrte darauf, zunächst noch einmal für einige Wochen daheim selbst ihren Blutdruck zu messen. Ich diskutierte das nicht mit ihr, da sie sich ja nicht in einem kritischen $\mathrm{Zu}$ - stand befand und manche Patienten sich am besten selbst überzeugen können. Dazu, so dachte ich, kann eine Blutdruckmessung in Eigenverantwortung sicherlich beitragen.

Nach einigen Wochen rief sie mich schließlich an, um über die Ergebnisse ihrer Bemühungen zu sprechen. Mit Inbrunst beteuerte sie: „Frau Doktor, die systolischen Werte waren immer gut, und auch die diabolischen Werte waren nur minimal erhöht!“

Dr. Michaela Rieke, Düsseldorf

\section{Verständigungsprobleme bei der Medikation}

Unter meinen Patienten war auch eine zunehmend demente Dame, bei der sich ein Pfleger einquartiert hatte, der sie versorgte. Wie viele Berufstätige aus dieser Branche kam der Mann aus Polen und sprach nur sehr gebrochen deutsch. Eines Tages kam er in die Praxis, um die planmäßig einzunehmenden Medikamente nachzubestellen. Auf "Madopah" und „Bisopol“ kam er noch, dann stockte er, überlegte - und bestellte schließlich „eine Abwassertablettäh“.

Dr. Herbert Zimmer, Freiburg 